\title{
Blood-Flow-Restriction Training: Validity of Pulse Oximetry to Assess Arterial Occlusion Pressure
}

\author{
Zhen Zeng, Christoph Centner, Albert Gollhofer, and Daniel König
}

\begin{abstract}
Purpose: Setting the optimal cuff pressure is a crucial part of prescribing blood-flow-restriction training. It is currently recommended to use percentages of each individual's arterial occlusion pressure, which is most accurately determined by Doppler ultrasound (DU). However, the practicality of this gold-standard method in daily training routine is limited due to high costs. An alternative solution is pulse oximetry (PO). The main purpose of this study was to evaluate validity between PO and DU measurements and to investigate whether sex has a potential influence on these variables. Methods: A total of 94 subjects were enrolled in the study. Participants were positioned in a supine position, and a 12-cm-wide cuff was applied in a counterbalanced order at the most proximal portion of the right upper and lower limbs. The cuff pressure was successively increased until pulse was no longer detected by DU and PO. Results: There were no significant differences between the DU and PO methods when measuring arterial occlusion pressure at the upper limb $(P=.308)$. However, both methods showed considerable disagreement for the lower limbs $(P=.001)$, which was evident in both men $(P=.028)$ and women $(P=.008)$. No sex differences were detected. Conclusions: PO is reasonably accurate to determine arterial occlusion pressure of the upper limbs. For lower limbs, PO does not seem to be a valid instrument when assessing the optimal cuff pressure for blood-flow-restriction interventions compared with DU.
\end{abstract}

Keywords: Doppler ultrasound, vascular occlusion, KAATSU, cuff pressure

The combination of physical exercise with a partial blood flow restriction (BFR) in the exercising extremity has gained increasing interest in both research settings and practical training applications. Previous investigations have demonstrated that low-load resistance training in combination with BFR promotes increases in muscle mass and strength to a similar extent as traditional high-load training. ${ }^{1-3}$ Besides cuff width ${ }^{4-6}$ and the duration of BFR, ${ }^{7}$ cuff pressure intensity is considered to be one of the most important determinants for optimal training adaptations $\mathrm{s}^{8,9}$ with both acute and chronic studies demonstrating pressure-dependent physiological responses. ${ }^{10-12}$

While some studies use the same absolute pressure across all individuals, ${ }^{13,14}$ setting an arbitrary absolute pressure does not necessarily restrict the same amount of blood flow for each individual and does thus not allow adequate standardization across subjects. With respect to relative pressure intensities, some studies have adjusted the applied cuff pressure on the lower limbs on the subjects' brachial blood pressure. ${ }^{15}$ This procedure is, however, questionable, because the brachial blood pressure does not necessarily explain substantial variance in the prediction of blood pressure in the lower limb. ${ }^{6}$ To provide an accurate and comparable degree of blood flow during BFR for each individual, it has been proposed to

(C) 2019 The Authors. Published by Human Kinetics, Inc. This is an Open Access article distributed under the terms of the Creative Commons Attribution License CC BY NC 4.0, which permits unrestricted noncommercial use, distribution, and reproduction in any medium, provided the original work is properly cited, the new use includes a link to the license, and any changes are indicated. See http:// creativecommons.org/licenses/by-nc/4.0. This license does not cover any third-party material which may appear with permission in the article. For commercial use, permission should be requested from Human Kinetics, Inc., through the Copyright Clearance Center (http://www.copyright.com).

Zeng is with the School of Sports Medicine and Health, Chengdu Sport University, Chengdu, China. Zeng, Centner, Gollhofer, and König are with the Dept of Sport and Sport Science, University of Freiburg, Freiburg, Germany. Centner (christoph. centner@sport.uni-freiburg.de) is corresponding author. apply pressure intensities relative to the pressure, which is needed to completely occlude arterial blood flow (arterial occlusion pressure $[\mathrm{AOP}])$.

The most frequently applied method to determine blood flow and thus AOP is the Doppler ultrasound (DU) technique. However, despite its high accuracy, the practicability of this gold-standard method $^{16,17}$ is limited, mainly owing to the limited availability of DU and the sum of the costs that arise with additional equipment. An alternative solution to assess changes in blood volume and pulse pressure is pulse oximetry (PO). The PO is a clinically established easy-to-use low-cost method. Implementing this method into BFR training regimes could therefore help to make BFR training more accessible for the population at large with the chance of being able to set the optimal cuff pressure without having a specialized training in applying DU technique.

Accordingly, an increasing number of studies have used PO to define the extent of BFR as well as assessing the AOP in BFR research. ${ }^{17-21}$ However, there is a lack of evidence regarding the accuracy of PO in determining both lower- and upper-limb AOP for BFR protocols.

Therefore, the main purpose of this study was to evaluate the validity between PO for measuring the AOP and hand-held DU as the current gold standard in the upper and lower limbs. As previous studies have reported substantial gender differences in limb circumference $^{22}$ and composition ${ }^{23}$ as well as oxygen dissociation curves, ${ }^{24}$ which in turn can affect PO readings and AOP, ${ }^{6}$ a secondary aim of this study was to evaluate whether gender has a potential influence on the AOP measurement.

\section{Methods}

\section{Subjects}

Based on the results of a power analysis (alpha $=.05$, power $=$ 0.9 , number of repeated observations $=2), 94$ (47 males and 
47 females) subjects (31.3 [12.7] y) volunteered to participate in the study. Before study initiation, test-retest reliability of the DU measurement $(\mathrm{N}=11)$ was assessed in an additional pilot project. The results demonstrated high reliability when reassessing the AOP to the nearest $5 \mathrm{~mm} \mathrm{Hg}$ after a 10-min resting period (intraclass correlation coefficient $>.9$ ). For this study, all participants were healthy and had no history of coagulation disorders including deep vein thrombosis. Further exclusion criteria were smoking, pregnancy, the presence of chronic degenerative diseases, uncontrolled hypertension, or medications affecting blood flow regulation. All participants gave written informed consent prior to participation. If subjects were eligible, anthropometric data including weight, height, brachial blood pressure, and limb circumference on the right arm and thigh were assessed. For assessing the circumference of the right arm, the distance between the acromion process and the olecranon was measured and a mark was made at $50 \%$ of the total length. In addition, the circumference of the right leg was determined at $25 \%$ of the femur length measured from the greater trochanter to the lateral epicondyle.

\section{Experimental Design}

To compare the accuracy of the DU and PO method, a repeatedmeasures, cross-over design was chosen. The AOP of each participant was determined in a random and counterbalanced fashion on both right upper and lower limbs with DU and PO, respectively. Before the AOP determination, participants were asked to rest in the supine position for 10 minutes. In addition, a rest period of $10 \mathrm{~min}$ utes was provided between the 4 measurements to ensure normalization of hemodynamics. ${ }^{25}$ All measurements were conducted in a quiet and temperature-controlled room $\left(22^{\circ} \mathrm{C}\left[1^{\circ} \mathrm{C}\right]\right)$. One experimenter completed all measurements to reduce interrater variability.

\section{Determination of AOP With Doppler Ultrasound}

For determining the AOP with the DU method, a 12-cm wide pneumatic nylon tourniquet (Zimmer Biomet, Warsaw, IN) was placed at the most proximal portion of the right arm $(50 \%$ of the length) or leg (25\% of the femur length). The cuff was attached with a snug fit ${ }^{5}$ by the same assessor. Subsequently, the posterior tibial artery or radial artery pulse was detected with a hand-held Doppler ultrasound (Handydop; Kranzbühler, Solingen, Germany). The cuff pressure was then gradually increased by $10 \mathrm{~mm} \mathrm{Hg}$ until a pulse was no longer detected. At this point, an arterial occlusion of $100 \%$ was assumed. The lowest pressure, at which the auditory signal was no longer detected, was documented and cuff pressure subsequently deflated.

\section{Determination of AOP With Pulse Oximeter}

With the cuff being at exactly the same position, a pulse oximeter (Zimmer Biomet) was placed at the index finger and first toe after cleaning the respective location with an alcoholic solution for skin disinfection. The cuff pressure was then stepwise increased by $10 \mathrm{~mm} \mathrm{Hg}$ until the signal of the PO indicated that periodic changes in blood volume could no longer be detected.

\section{Statistical Analysis}

Statistical analyses were conducted with SPSS (version 24.0; IBM, Armonk, NY) and the alpha level was set to $P<.05$. After checking for normal distribution of all variables, a paired $t$ test was used to investigate the difference between DU and PO.

The distribution of the differences was plotted using a BlandAltman plot. The Bland-Altman plot demonstrated the degree of agreement between the 2 investigated methods. All analyses were conducted for females and males, as well as for the mixed population. For all data, an outlier analysis (mean [3 SD]) was performed and 5 subjects were excluded from the analysis. Statistical analysis of the AOP difference was completed after the removal of 5 paired measurements as outliers. All data are presented as mean (SD) unless otherwise stated.

\section{Results}

Table 1 summarizes the subjects' descriptive and anthropometric characteristics. The AOP differences for all measurements are shown in Figure 1.

All subjects completed the investigation and no dropouts were reported. However, due to invalid PO readings, data from $n=9$ lower limbs and $n=4$ upper limbs were not included in the data analysis. The results indicated that there were no significant differences between the DU and PO method when measuring the AOP at the upper limb (1.52 [13.94] $\mathrm{mm} \mathrm{Hg}, P=.308)$. However, after calculation of a paired $t$ test, statistically significant differences were detected when determining the AOP at the lower limb (8.46 [21.15] mm Hg, $P=.001)$.

In a subsequent subgroup analysis, we discriminated for gender and found that male (Figure 2[A]) and female subjects (Figure 3[A]) showed a nonsignificant difference in means of -1.17 (12.68) $\mathrm{mm} \mathrm{Hg} \quad(P=.533)$ and 4.48 (14.78) $\mathrm{mm} \quad \mathrm{Hg}$ $(P=.057)$ for upper limbs, respectively. However, significant differences in lower limbs have been identified with 6.53 (18.80) $\mathrm{mm} \mathrm{Hg}(P=.028)$ and 10.59 (23.52) $\mathrm{mm} \mathrm{Hg}(P=.008)$ for males (Figure 2[B]) and females (Figure 3[B]), respectively.

\section{Discussion}

This study aimed to compare the accuracy of the DU and PO methods in determining AOP. The main findings indicated that there was no significant difference between both methods when the AOP was measured at the upper limb. Although the statistical tests for differences did not reach significance in the upper limbs, this does not necessarily indicate a good agreement between the methods. However, we observed a reasonable agreement between PO and DU with most observations being located within a range of $15 \mathrm{~mm} \mathrm{Hg}$ around the mean difference. ${ }^{25}$ Setting relative pressures during BFR training interventions in upper limbs further decreases the observed AOP differences between $\mathrm{PO}$ and DU in practice. When measuring the AOP at the lower limb, the PO method demonstrated considerable differences, compared with the DU as the current gold standard. This is

Table 1 Descriptive Characteristics for Participants, Mean (SD)

\begin{tabular}{lllcccr}
\hline Group & N & Age, $\mathbf{y}$ & Weight, $\mathbf{k g}$ & Height, cm & Arm circumference, cm & Thigh circumference, cm \\
\hline Female & 47 & $29(11)$ & $62.2(9.8)$ & $165.6(5.5)$ & $28.0(2.7)$ & $54.9(4.0)$ \\
Male & 47 & $34(14)$ & $82.0(12.5)$ & $181.4(6.5)$ & $31.8(2.5)$ & $55.8(4.2)$ \\
Both sexes & 94 & $31(13)$ & $72.1(15.0)$ & $173.5(9.9)$ & $29.9(3.2)$ & $55.4(4.1)$ \\
\hline
\end{tabular}



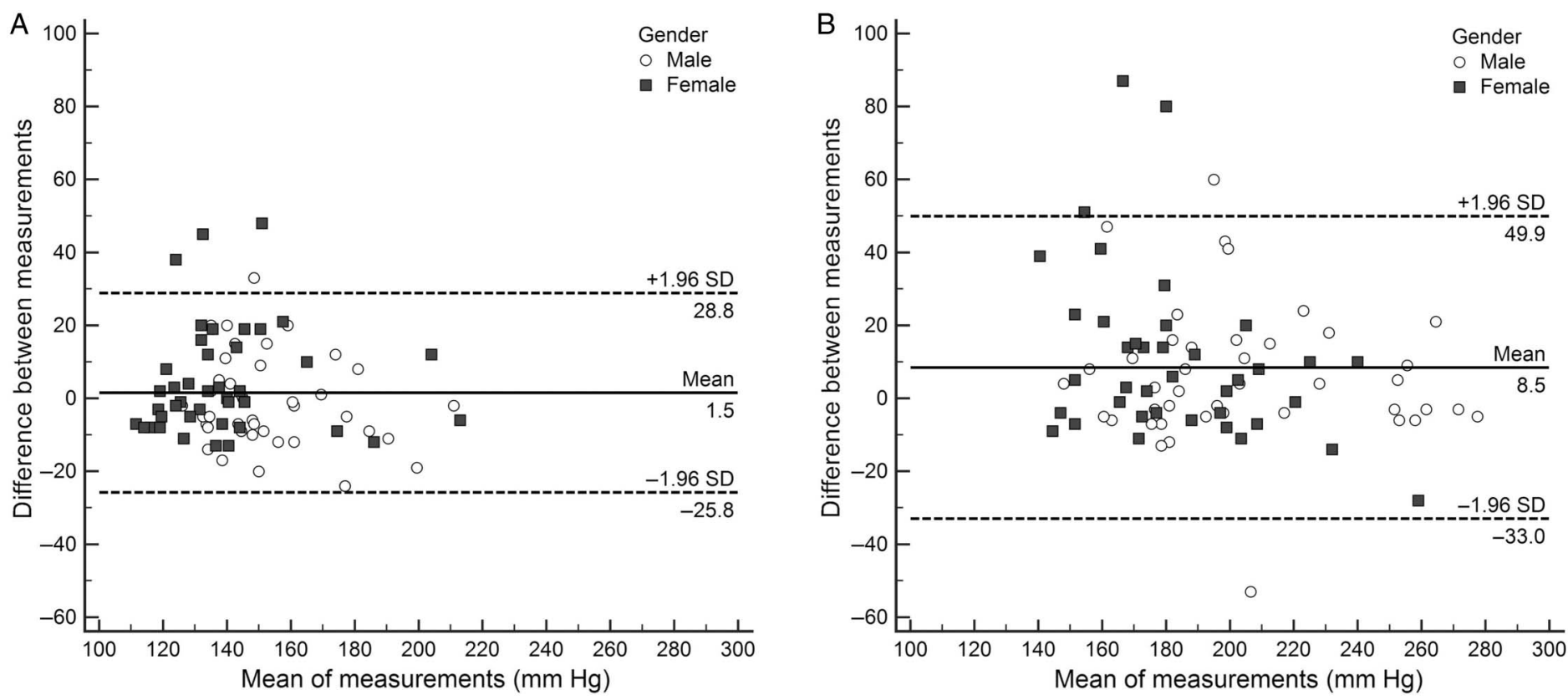

Figure 1 - Bland-Altman plot of the differences in arterial occlusion pressure between Doppler ultrasound and pulse oximetry for upper limbs (A) and lower limbs (B) in male (circles) and female (squares) participants.

also highlighted by the Bland-Altman plot revealing substantial disagreement.

Currently available oximeters use 2 light emitting electrodes that emit red (660 nm wavelength) and near-infrared (940 nm wavelength) light through the region of interest. ${ }^{26-28}$ The light absorbance is then measured by a photodiode. Oxyhemoglobin and reduced hemoglobin have different absorption spectra, and this allows to determine oxygen saturation and thus also pulse, because the blood volume in the arteries (and thus light absorption) fluctuates with the cardiac cycle. ${ }^{26}$ In general, the rate of absorption can be influenced by several factors including probe positioning, cold temperature, skin pigmentation, excessive movement, poor perfusion, ${ }^{26}$ or fingernail polish. ${ }^{26,29}$ The fact that there is generally an increased epidermal thickness at the sole of the foot ${ }^{30}$ might help explain the substantial levels of inaccuracy of the PO when measuring the AOP at the lower limb compared with the upper limb. In addition, the blood flow in both feet and hands seems to be greatly dependent on temperature changes. ${ }^{31,32}$ However, it appears that blood flow in the hand is considerably greater compared with that in the foot when temperatures lower than $32^{\circ} \mathrm{C}$ are applied. ${ }^{31,32}$ Thus, it might be argued that the accuracy of the $\mathrm{PO}$ readings at the foot are more biased than measurements at the hand, due to temperature-induced decreases in perfusion.

Previous investigations have noted that nail polish negatively affects PO readings, reducing its values by up to $10 \% .{ }^{29,33} \mathrm{In}$ particular, black and brown polish seem to induce the greatest bias. ${ }^{34}$ In this study, we did not assess nail polish as a covariate and can thus not eliminate the influence of this variable. Furthermore, no conclusions can be drawn about the level of AOP in a sitting or standing position. A study by Sieljacks et $\mathrm{al}^{5}$ indicated that body position influences lower limb AOP, with significantly higher values in a sitting position. Therefore, future studies need to determine whether the PO may give more accurate readings when measuring the AOP in different body positions. Because of the novelty of our results and the corresponding research question, limited comparisons can be made between other studies. According to the AOP determination, previous investigations ${ }^{35}$ have reported similar pressures $(224 \mathrm{~mm} \mathrm{Hg})$, which are comparable with an AOP of $207 \mathrm{~mm} \mathrm{Hg}$ that was found for the male subjects in this study. The observed differences in AOP $(\sim 17 \mathrm{~mm} \mathrm{Hg})$ might likely be attributable to slight variations in cuff width, which was $10.5 \mathrm{~cm}$ in the study by Clarkson et al. ${ }^{35}$ This is in accordance with evidence from the literature showing that higher pressure intensities are required with narrow cuffs compared with wide cuffs. ${ }^{36}$

\section{Practical Applications}

Setting a relative cuff pressure is of essential importance when training with partial vascular occlusion. This is underpinned by the fact that both acute and chronic studies have demonstrated cuff pressure-dependent changes in electromyography amplitude. ${ }^{10-12}$ Loenneke et $\mathrm{al}^{11}$ and Fatela et $\mathrm{al},{ }^{10}$ for instance, reported that muscle activation changes with a function of cuff pressure intensity, with greater pressures inducing greater electromyography activities. In addition, evidence from a longitudinal study suggests that a higher occlusion pressure increases muscular hypertrophy at lower training intensities. ${ }^{12}$ This highlights the necessity to individually determine the AOP before engaging in BFR training. Besides avoiding arbitrary pressures, investigators should aim to assess AOP during each training visit and not just once or on a weekly basis, ${ }^{3}$ because AOP has been shown to underlie diurnal variations. $^{37}$ In both research and practice, the PO method can make the AOP assessment during BFR training more accessible and easier to use, even without being specially trained in ultrasound technique. Although our findings suggest that the PO method is a sufficiently valid tool for determining the AOP in the upper limbs, no acceptable accuracy was found for the lower limbs. It is well-known that poor signal quality during $\mathrm{PO}$ assessment can result in invalid readings, ${ }^{38}$ which further limits the applicability of PO in 


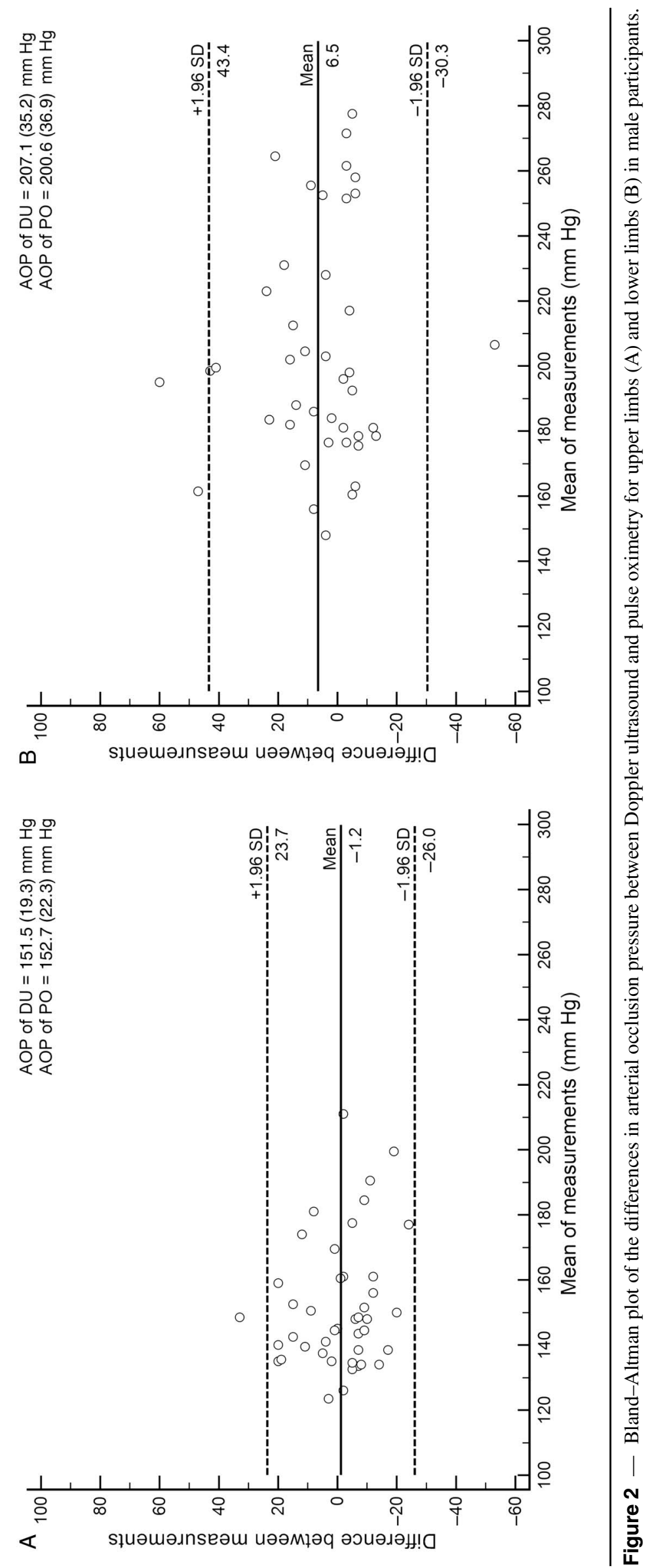

IJSPP Vol. 14, No. 10, 2019 


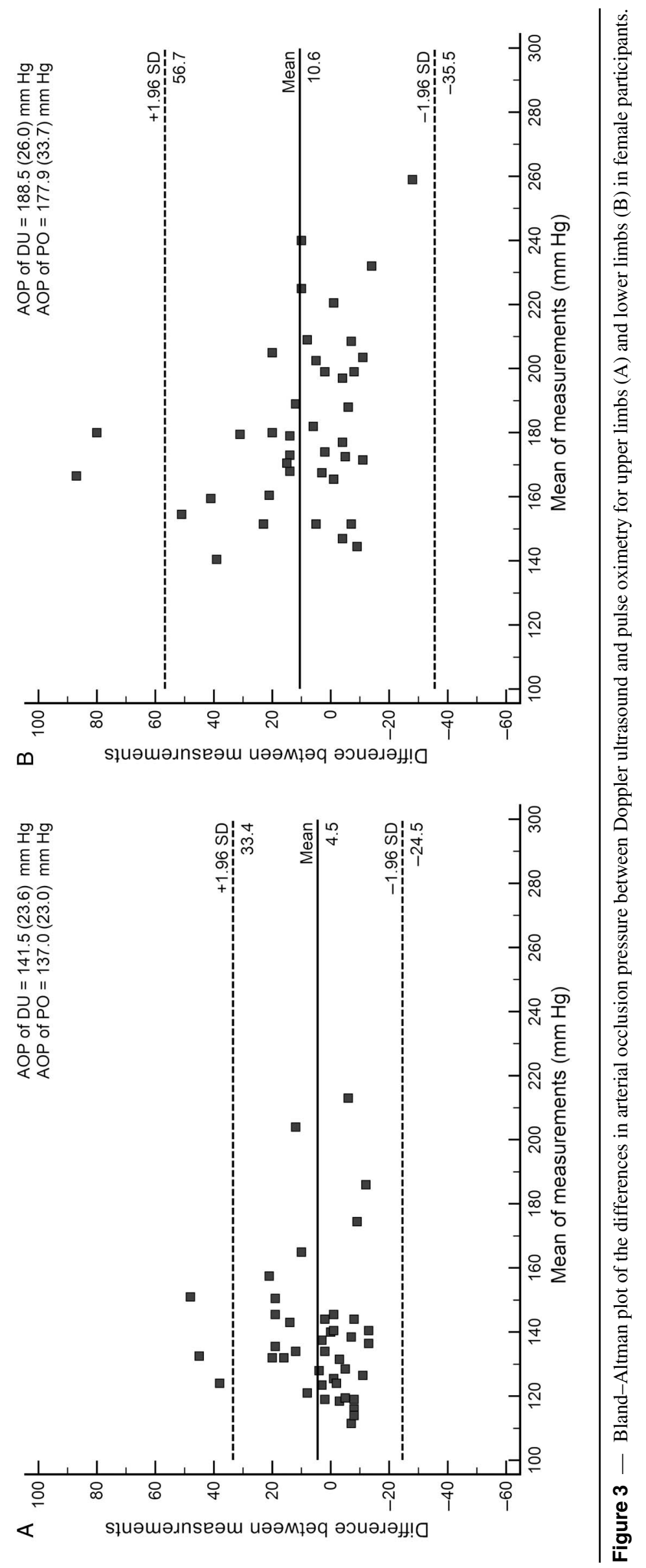


research and practice. The limits of agreement might have also been taken into account when setting the cuff pressure during both acute and chronic BFR interventions. Even a slight disagreement in the procedure of AOP assessment with PO might have an immediate effect on training adaptations and physiological responses.

In this matter, the aspect of safety considerations needs to be mentioned. Especially, in clinical settings, it is particularly necessary to cautiously apply PO, because various diseases, including Raynaud's disease, scleroderma ${ }^{39}$ or jaundice, ${ }^{40}$ might interfere with pulse oximetric detection.

\section{Conclusions}

From these data, it can be concluded that PO is a reasonably accurate method for determining AOP for the upper limbs. For the lower limbs, however, PO seems to be less accurate when aiming for standardized BFR in BFR exercise interventions compared with DU as the current gold standard.

\section{Acknowledgment}

The authors thank the subjects who voluntarily participated in this study. None of the authors declare any conflict of interest.

\section{References}

1. Karabulut M, Abe T, Sato Y, Bemben MG. The effects of lowintensity resistance training with vascular restriction on leg muscle strength in older men. Eur J Appl Physiol. 2010;108(1):147-155. PubMed ID: 19760431 doi:10.1007/s00421-009-1204-5

2. Lixandrao ME, Ugrinowitsch C, Berton R, et al. Magnitude of muscle strength and mass adaptations between high-load resistance training versus low-load resistance training associated with bloodflow restriction: a systematic review and meta-analysis. Sports Med. 2018;48(2):361-378. PubMed ID: 29043659 doi:10.1007/s40279017-0795-y

3. Vechin FC, Libardi CA, Conceicao MS, et al. Comparisons between low-intensity resistance training with blood flow restriction and highintensity resistance training on quadriceps muscle mass and strength in elderly. J Strength Cond Res. 2015;29(4):1071-1076. PubMed ID: 25264670 doi:10.1519/JSC.0000000000000703

4. Loenneke JP, Fahs CA, Rossow LM, et al. Blood flow restriction pressure recommendations: a tale of two cuffs. Front Physiol. 2013;4:249. PubMed ID: 24058346

5. Sieljacks P, Knudsen L, Wernbom M, Vissing K. Body position influences arterial occlusion pressure: implications for the standardization of pressure during blood flow restricted exercise. Eur J Appl Physiol. 2018;118(2):303-312. PubMed ID: 29196847 doi:10.1007/ s00421-017-3770-2

6. Loenneke JP, Fahs CA, Rossow LM, et al. Effects of cuff width on arterial occlusion: implications for blood flow restricted exercise. Eur J Appl Physiol. 2012;112(8):2903-2912. PubMed ID: 22143843 doi:10.1007/s00421-011-2266-8

7. Dankel SJ, Buckner SL, Jessee MB, et al. Post-exercise blood flow restriction attenuates muscle hypertrophy. Eur J Appl Physiol. 2016; 116(10):1955-1963. PubMed ID: 27480315 doi:10.1007/s00421016-3447-2

8. Loenneke JP, Thiebaud RS, Abe T, Bemben MG. Blood flow restriction pressure recommendations: the hormesis hypothesis. Med Hypotheses. 2014;82(5):623-626. PubMed ID: 24636784 doi:10. 1016/j.mehy.2014.02.023
9. Counts BR, Dankel SJ, Barnett BE, et al. Influence of relative blood flow restriction pressure on muscle activation and muscle adaptation. Muscle Nerve. 2016;53(3):438-445. PubMed ID: 26137897 doi:10. 1002/mus. 24756

10. Fatela P, Reis JF, Mendonca GV, Avela J, Mil-Homens P. Acute effects of exercise under different levels of blood-flow restriction on muscle activation and fatigue. Eur J Appl Physiol. 2016;116(5): 985-995. PubMed ID: 27017495 doi:10.1007/s00421-016-3359-1

11. Loenneke JP, Kim D, Fahs CA, et al. Effects of exercise with and without different degrees of blood flow restriction on torque and muscle activation. Muscle Nerve. 2015;51(5):713-721. PubMed ID: 25187395 doi:10.1002/mus. 24448

12. Lixandrao ME, Ugrinowitsch C, Laurentino G, et al. Effects of exercise intensity and occlusion pressure after 12 weeks of resistance training with blood-flow restriction. Eur J Appl Physiol. 2015; 115(12):2471-2480. PubMed ID: 26323350 doi:10.1007/s00421015-3253-2

13. Renzi CP, Tanaka H, Sugawara J. Effects of leg blood flow restriction during walking on cardiovascular function. Med Sci Sports Exerc. 2010;42(4):726-732. PubMed ID: 19952840 doi:10.1249/MSS. 0b013e3181bdb454

14. Yasuda T, Abe T, Sato Y, et al. Muscle fiber cross-sectional area is increased after two weeks of twice daily KAATSU-resistance training. Int J KAATSU Training Res. 2005;1(2):65-70. doi:10.3806/ ijktr.1.65

15. Clark BC, Manini TM, Hoffman RL, et al. Relative safety of 4 weeks of blood flow-restricted resistance exercise in young, healthy adults. Scand J Med Sci Sports. 2011;21(5):653-662. PubMed ID: 21917016 doi:10.1111/j.1600-0838.2010.01100.x

16. Graham B, Breault MJ, McEwen JA, McGraw RW. Occlusion of arterial flow in the extremities at subsystolic pressures through the use of wide tourniquet cuffs. Clin Orthop Relat Res. 1993;286: 257-261.

17. Masri BA, Day B, Younger ASE, Jeyasurya J. Technique for measuring limb occlusion pressure that facilitates personalized tourniquet systems: a randomized trial. J Med Biol Eng. 2016;36(5):644650. PubMed ID: 27853415 doi:10.1007/s40846-016-0173-5

18. Fry CS, Glynn EL, Drummond MJ, et al. Blood flow restriction exercise stimulates mTORC1 signaling and muscle protein synthesis in older men. J Appl Physiol. 2010;108(5):1199-1209. doi:10.1152/ japplphysiol.01266.2009

19. Clarkson MJ, Fraser SF, Bennett PN, McMahon LP, Brumby C, Warmington SA. Efficacy of blood flow restriction exercise during dialysis for end stage kidney disease patients: protocol of a randomised controlled trial. BMC Nephrology. 2017; 18:294. PubMed ID: 28893206 doi:10.1186/s12882-017-0713-4

20. Hollander DB, Reeves GV, Clavier JD, Francois MR, Thomas C, Kraemer RR. Partial occlusion during resistance exercise alters effort sense and pain. J Strength Cond Res. 2010;24(1):235-243. PubMed ID: 19935100 doi:10.1519/JSC.0b013e3181c7badf

21. Kraemer RR, Goldfarb AH, Reeves GV, Meachum WA, Castracane VD. Effects of partial vascular occlusion on irisin responses to loaded muscle contractions. Appl Physiol Nutr Metab. 2016;41(3):332-334. PubMed ID: 26859524 doi:10.1139/apnm-2015-0464

22. Benitez Brito N, Suarez Llanos JP, Fuentes Ferrer M, et al. Relationship between mid-upper arm circumference and body mass index in inpatients. PLoS ONE. 2016;11(8):e0160480. PubMed ID: 27494612 doi:10.1371/journal.pone.0160480

23. Ethun K. Chapter 9-Sex and gender differences in body composition, lipid metabolism, and glucose regulation. In: Neigh GN, Mitzelfelt MM, eds. Sex Differences in Physiology. Boston, MA: Academic Press; 2016:145-165. 
24. Boning D, Draude W, Trost F, Meier U. Interrelation between Bohr and temperature effects on the oxygen dissociation curve in men and women. Respir Physiol. 1978;34(2):195-207. PubMed ID: 705080 doi:10.1016/0034-5687(78)90028-2

25. Laurentino GC, Loenneke JP, Mouser JG, et al. Validity of the handheld Doppler to determine lower-limb blood flow restriction pressure for exercise protocols [published online ahead of print June 14, 2018]. J Strength Cond Res.

26. Chan ED, Chan MM, Chan MM. Pulse oximetry: Understanding its basic principles facilitates appreciation of its limitations. Respir Med. 2013;107(6):789-799. PubMed ID: 23490227 doi:10.1016/j.rmed. 2013.02.004

27. Jubran A. Pulse oximetry. Crit Care. 1999;3(2):R11-R17. PubMed ID: 11094477 doi:10.1186/cc341

28. Jubran A. Pulse oximetry. Crit Care. 2015;19:272. PubMed ID: 26179876 doi:10.1186/s13054-015-0984-8

29. Cote CJ, Goldstein EA, Fuchsman WH, Hoaglin DC. The effect of nail polish on pulse oximetry. Anesth Analg. 1988;67(7):683-686. PubMed ID: 3382042

30. Lee Y, Hwang K. Skin thickness of Korean adults. Surg Radiol Anat. 2002;24(3-4):183-189.

31. Allwood MJ, Burry HS. The effect of local temperature on blood flow in the human foot. $J$ Physiol. 1954;124(2):345-357. PubMed ID: 13175136 doi:10.1113/jphysiol.1954.sp005112

32. Spealman CR. Effect of ambient air temperature and of hand temperature on blood flow in hands. Am J Physiol. 1945;145: 218-222. PubMed ID: 21009263 doi:10.1152/ajplegacy.1945.145. 2.218
33. Hakverdioglu Yont G, Akin Korhan E, Dizer B. The effect of nail polish on pulse oximetry readings. Intensive Crit Care Nurs. 2014;30(2): 111-115. PubMed ID: 24054173 doi:10.1016/j.iccn.2013.08.003

34. Chan MM, Chan MM, Chan ED. What is the effect of fingernail polish on pulse oximetry? Chest. 2003;123(6):2163-2164. PubMed ID: 12796214 doi:10.1378/chest.123.6.2163

35. Clarkson MJ, Conway L, Warmington SA. Blood flow restriction walking and physical function in older adults: a randomized control trial. J Sci Med Sport. 2017;20(12):1041-1046. PubMed ID: 28483555 doi:10.1016/j.jsams.2017.04.012

36. Crenshaw AG, Hargens AR, Gershuni DH, Rydevik B. Wide tourniquet cuffs more effective at lower inflation pressures. Acta Orthop Scand. 1988;59(4):447-451. PubMed ID: 3421083 doi:10.3109/ 17453678809149401

37. Ingram JW, Dankel SJ, Buckner SL, et al. The influence of time on determining blood flow restriction pressure. J Sci Med Sport. 2017;20(8):777-780. PubMed ID: 28131507 doi:10.1016/j.jsams. 2016.11.013

38. Webb RK, Ralston AC, Runciman WB. Potential errors in pulse oximetry. II. Effects of changes in saturation and signal quality. Anaesthesia. 1991;46(3):207-212. PubMed ID: 2014899 doi:10. 1111/j.1365-2044.1991.tb09411.x

39. Garg R, Verma S. Pulse oximetry in scleroderma patients: concerns Acta Anaesthesiol Scand. 2008;52(9):1303-1304. PubMed ID: 18823473 doi:10.1111/j.1399-6576.2008.01720.x

40. Nilles K, Subramanian R. Excessive hyperbilirubinemia interferes with pulse oximetric detection of hypoxemia in decompensated cirrhosis. Crit Care Med. 2012;40(12):U321-U322. 